\title{
Showing 'digital' objects in web-based video chats as a collaborative achievement
}

\author{
Laura Rosenbaun and Christian Licoppe \\ University of Haifa, Israel ${ }^{1}$ / Telecom ParisTech, France
}

\begin{abstract}
Showing material objects by bringing them to the camera or turning the camera toward them are pervasive practices in domestic and recreational videomediated communication (VMC). We here discuss a set of specific showing practices characteristic of digitally embedded video-mediated settings, which may be called 'digital showings'. These involve participants' collaboration to retrieve a digital object so as to ensure a shared perceptual experience on screen of said object. We draw on data from multiparty Google Hangouts On Air (HOAs) to show that while digital and material showings share an overall sequential organization, the former display the emergence of unique collaborative practices that at times become collective performances of computer literacy. We focus on three instances of digital showings: (a) screenshares of pictures - showing an image by sharing one's screen; (b) screenshares of videos - showing a running video by sharing one's screen; and (c) link-share showings - showing by sharing the link to a showable content that may be independently retrieved while experienced jointly.
\end{abstract}

Keywords: Google Hangouts On Air, video-mediated conversation, telepresence, conversation analysis, sequential organisation, digital showings

\section{Introduction}

Showing sequences in which co-participants make accessible to others a given behavior, person, or detail in their environment, are a common practice in domestic and recreational video-mediated communication (VMC). We use the notion of 'showing' as an umbrella term to gloss a set of practices through which some particular feature of the environment that is initially unequally available in perceptual terms to all co-participants, is made into a relevant 'showable' and manipulated so

1. Currently at Human-Machine Interaction at General Motors, Warren, MI. 
as to be reshaped into a joint focus of attention. Leveling the visual field and redressing the inequality in perceptual access is a core feature of 'showings'. It is this manipulative work or 'placing' objects in a common perceptual field (Clark 2003) and the access imbalance it indexes what differentiates 'showing' from 'pointing'. In the latter, the relevant feature (the 'pointable') is often presumed to be available in the environment (Kita 2003). However, as interactional achievements, pointing and showing practices display a resemblance: (a) both are embodied, multimodal practices in which material features in the environment are turned into interactional resources; (b) even in pointing, some orientation work is often needed to ensure the pointed feature becomes a joint focus of attention; and (c) the embodied work involved in pointing (Goodwin 2003; Heath \& Von Lehn 2004), as well as that involved in showing (Licoppe \& Morel, 2014), are co-emergent and mutually elaborated along the concurrent talk-in-interaction.

Because co-presence implies that many features of the environment are mutually accessible to co-participants, pointing practices are more common and have been studied more extensively than 'showings'. One type of co-present 'showing' that has nevertheless received some attention, although not described as such, involves mobile phones (Weilenmann \& Larsson 2001; Aaltonen et al. 2014; Robles et al. forthcoming). Here, the small size screen implies that it has to be 'shown' to a co-participant to be assessed in a proper way. One understands easily why VMC offers a congenial setting for showings. Video-mediated settings are paradigmatic examples of 'fragmented ecologies' (Luff et al. 2003), i.e. settings in which part of participants' environments is not perceptually (and especially visually) available to others. 'Showings' of previously invisible features in one's environment can then be accomplished either by turning the camera toward a given relevant feature (Licoppe \& Morel 2014), or by bringing a given object to the camera. In mobile video communication, it has been noted that a considerable percentage of video conversations revolves around one of the participants showing something to the other (O'Hara, Black, \& Lipson 2006). In order to effectively create a showing, users need to attend to the image they display on screen in close coordination with the ongoing talk-in-interaction. As such, showing sequences can adequately be described as 'video-in-interaction' (Licoppe \& Morel 2012).

The point of this communication is to provide evidence for a set of practices that could be characterized as a specific type of showing sequence, which we call 'digital showings'. In this type of showing, what participants put on display is a digital object rather than a physical or material one, emphasizing both the digital and mediated nature of the interaction and the embeddedness of web-based video communication within an infrastructure of digital services and affordances. In the Human Computer Interaction (HCI) community, this is often described as a kind of 'sharing' (as in screen sharing). However, such practices may also be glossed as 
'showings' since what is at play is the making a given digital object into a mutually shared focus of attention. Moreover, as we will argue, both material and digital showings share a three-part sequential organization: (a) preliminary work combining a reference to something that can be seen as a 'showable', which projects a showing, and the sequential implications that constitute a preface and frame the way the 'showable' should be seen. Such preliminary work enacts interactiongenerated categories (Watson 1997) such as 'show-er(s)' and 'viewer(s)', each with asymmetric capacities, rights, and obligations; (b) the showing itself, qua manipulation of the 'showable'; (c) some reception of the showable, displaying two distinct concerns, that one can see the showable, and that one can see it as it was framed. Although we will argue that the work involved in digital showings follows the same sequential organization as material showings, it also differs in its complexity, and in the collaboration patterns involved.

Our data consist of recording of interactions from public and multiparty video-mediated streams broadcast live for recreational and social purposes. Recreational synchronous VMC in public contexts appears to share characteristics from both domestic videoconferencing practices and the public broadcasting sphere. On the one hand, participants engage in activities previously discussed in the literature on domestic VMC, such as gossiping, and personal updates, among others (e.g. Buhler, Neustaedter, \& Hillman 2013). At the same time, characteristics from interactive TV such as viewers' participation through live text commentaries (LTCs) (Chovanec \& Novaka 2010) are also observed. Consequently, similarly to other broadcasting practices, interactions become public affairs, acquiring a multilayered dimension: individuals are engaged with friends through the visual/ aural feeds, with viewers through LTCs, and with a broad sense of an audience (Rosenbaun, Rafaeli \& Kurzon 2016a, 2016b).

Apart from online and offline friends, users engage with a perceived audience, or their 'imagined audience' (Litt 2012). This notion highlights users' perceptions of who their viewers may be and how their behavior may need to be adjusted accordingly. In our examples, the audience is constituted by viewers interacting through LTCs, non-interacting viewers, and potential future viewers in case the Hangout is archived in the host's YouTube channel. Since interactions are entirely accomplished through and dependent on the medium, mediation itself at times comes to the fore and becomes a central interactional resource (Rintel 2013). At other times, affordances are simply ignored since, as it has long being noted, users will not interact multimodally just because a system supports multimodality (Oviatt 1999). In fact, even when the video feed is on and users are visually available, the salience of the visual modality is not a given (Heath \& Luff 1992, Rosenbaun et al. 2016a). 


\section{Hangouts On Air}

We draw on multiparty video streams data from an application called Hangouts On Air (HOAs) or Hangouts. This application was launched by Google in 2011 as another attempt by that corporation to support social networks. One of the novel features supported by this application is that it combines video chat with a conference feature. While free video tools have been available through several services, it was this multiparty video conferencing feature that set this platform apart. Furthermore, the application also allowed broadcasting through a YouTube channel where anyone can watch live. The application could also automatically create a recording of the interactions and upload them to the host's YouTube channel. HOAs support interactions of up to 10 participants (up to 15 for some selected users). Figure 1 below shows the standard HOA screen configuration, in which all participants appear in the thumbnail strip at the bottom, the current speaker takes the main video window, ${ }^{2}$ and the LTCs posted by viewers appear in the messaging window on the right.
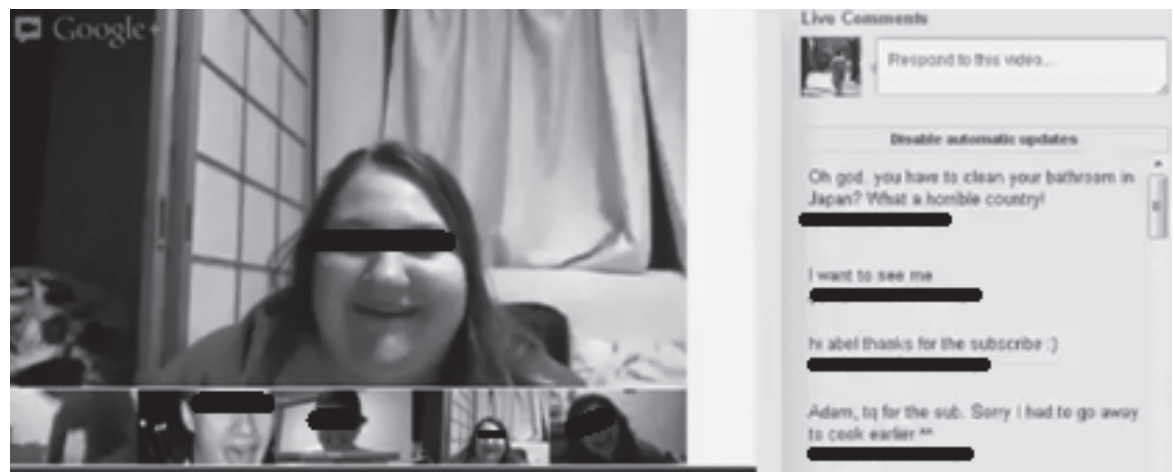

Figure 1. Screenshot of Hangout On Air

Our data were gathered between 2012 and 2013 using Camtasia Studio 8 software. 20 interactions were recorded, consisting of approximately 12 hours and 102 participants. From our observations, HOAs are organized by members who know one another from non-mediated environments or who have hung out in the past and agree to broadcast live through YouTube. Interactions can go on for several hours and topics of discussion switch freely around a given subject or broad shared interest. In addition, there is a central participant acting as the host who leads the flow

2. This feature is activated by the sound picked by the microphone or can be set by the Hangout host. See Rosenbaun et al. (2016). 
of the interaction. Different Hangouts range from more to less amateur in terms of broadcasting experience and skills, pre-set agenda, use of logos, and quality of technical equipment, among others. In all cases, however, participants must agree beforehand to make their interactions public, which implies an understanding of the potential presence of an audience. Due to the public nature of HOAs, informed consent was not used for this study following the concept of 'contextual integrity' proposed by Nissenbaum (2009) and upheld by the ethics guidelines of the Association of Internet Researchers. This concept helps us to determine contested notions such as public and private, and is a reminder that "what people care most about is not simply restricting the flow of information but ensuring that it flows appropriately" (p. 2). To this end, all participants' names, names given to their Hangouts, and information on geographical locations have been anonymized. All images of participants in talking head format have been censored.

We take a conversation analytic approach to studying showing sequences and how participants make use of resources to organize action and convey meaning (Goodwin 2000a, 2000b, 2013; Heath \& Luff 1991; Mondada 2011, 2013). Except for our last example, LTCs - comments posted by viewers - are not included in the transcript as they fall outside the scope of this study. Our analysis focuses on three examples. The first one consists of a group discussing allegedly hidden images in the video game they play. The second example is part of an interaction about cars, bikes, and racing. The last one belongs to a group interested in anime and manga.

\subsection{Example 1: The collaborative screen-shares of digital images}

We now elaborate on the sequential organization of digital showings, and the resources available to participants during such interactional projects. This Hangout group has been discussing hidden images that can be identified in certain parts of a video game (Princess Fantasy Faire). Participants are engaged in commenting whether the various characters that are supposedly depicted (e.g. the Yoda, Mickey Mouse) can actually be identified. When we enter the action, ELLIOT and COSMO are leading the interaction describing the spots where people need to look at in order to find a given image (section not transcribed). Figure 2 below shows the thumbnail image with all participants. TUCK, in the middle, is the Hangout host.

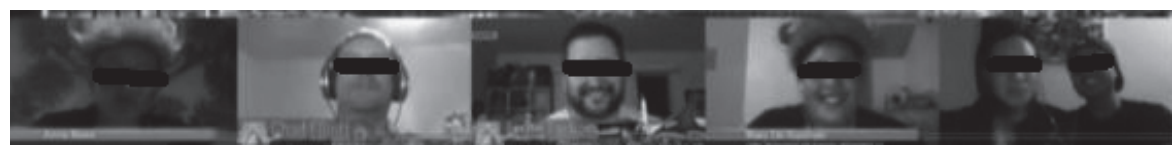

Figure 2. BLUE, ELLIOT, TUCK, GIRL, and ZELMA-COSMO 
TUCK has apparently been looking for examples of images to put on display. At the beginning of Extract 1, TUCK signals he has found a possible candidate. His turn is designed as an occasioned announcement, with a "oh" change-of-state token in initial position (Heritage 1984), and a subsequent announcement (Line 1). The self-repair in the announcement, from "I found some(thing)" to "I found one" displays a strong orientation toward framing what he has found as a candidate image.

ELLIOT orients toward the announcement with a clarification check (Line 4) and treats TUCK's next turn "on Milesway" (Line 5) as an inadequate response, upgrading the check to a question with a candidate answer (Line 6). Indeed "on Milesway" provides a sense of 'where' the image can be found and can be understood as a continuation of TUCK's announcement in line 3. This indexicality of location points to the 'digitality' of the image itself: it can be found online, and by mentioning its location, TUCK makes the referred-to image accessible to all the other participants.

\section{Extract 1.} TUCK: Oh [ I found some- ((TUCK brings hand to chin and moves closer
to monitor. Main window)) $\quad$ Image 1.1
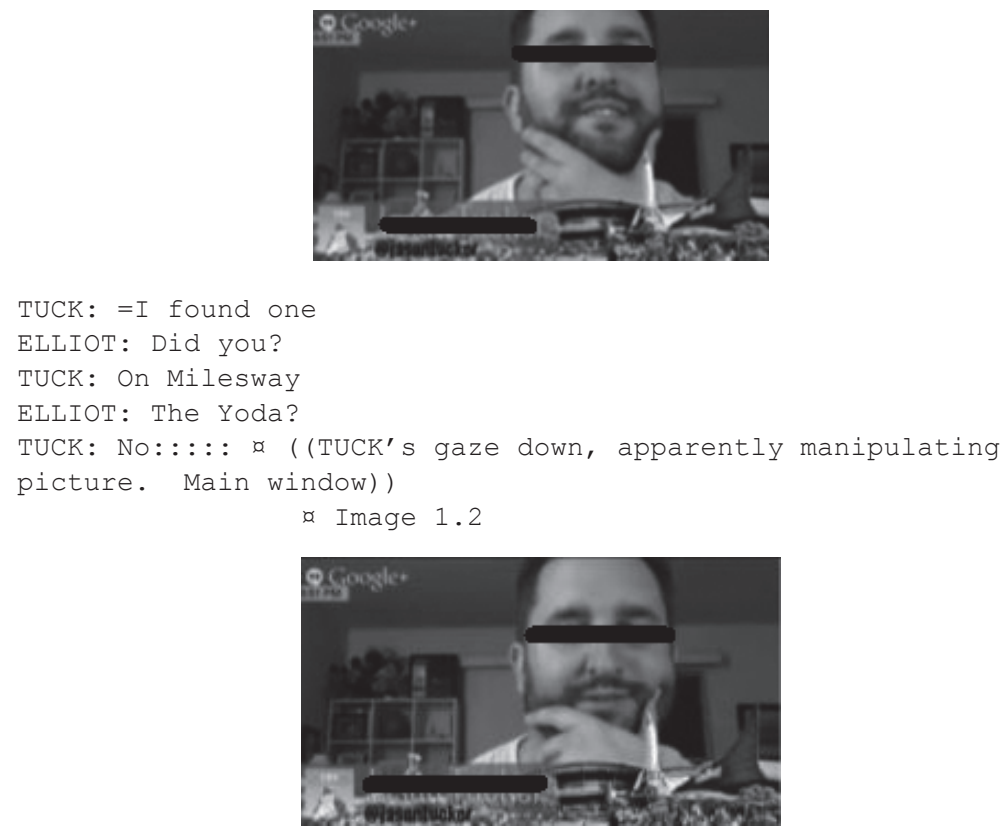

9 ELLIOT: Oh OK

10 TUCK: But you guys've probably seen this one right? ((TUCK's screenshare. Main 
11 window))

a Image 1.3

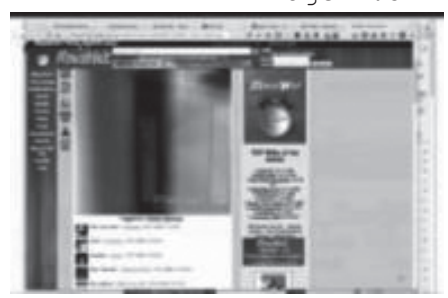

12

13

ZELMA: What is $=$ a

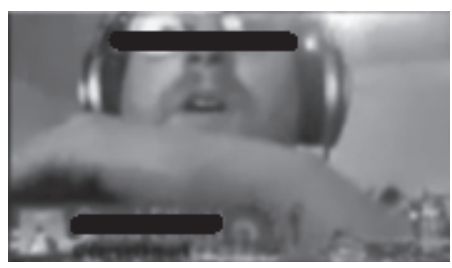

14 ELLIOT: I see the (Mickey) a ( (COSMO and ZELMA approach monitor. Thumbnail))

a Image 1.5

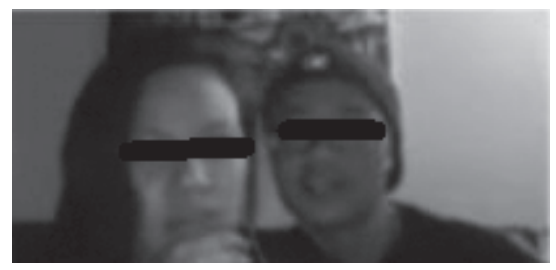

16 ZELMA: (.) Yeah: : : [ ( ) ((laughter))

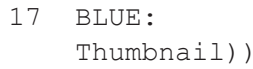

[That one- ((BLUE approaches monitor.

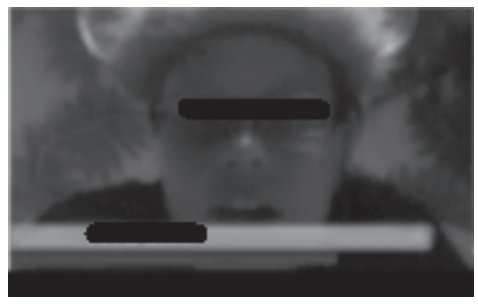

a Image 1.6

19 BLUE: =OK so that one was the one that I was trying to find but I actually found a

20 different one.. is that in Princess Fantasy Faire?

21 ZELMA: That's in [( ) Music Box

22 TUCK: [Yeah: : : :

23 BLUE: That's not the one I found in The Music Box 
24 COSMO: Uuuuhhh: : :

25 TUCK: ((signaling trouble)) Uh Oh ..

26 BLUE: ((exaggerated tone marking trouble)) Oohh[hhh: : : : : :

27 COSMO:

28 =Baaa: : :ng!! ॠ ((TUCK stops the screenshare) )

29 [SECTION NOT TRANSCRIBED. TUCK PRODUCES A FAILED SHOWING]

After responding to ELLIOT's check, TUCK also shares the image on screen, displaying it into their joint interactional space or "cooperative transformation zone" (Goodwin 2013), thus properly 'showing' it. The showing itself is preceded by a turn-at-talk, "but you guys've probably seen this one", which prefaces the visual event and evidences the public nature of the image. The deictic expression "this one" ties the talk both to the visual event to come (this one which will appear visually on screen) and anaphorically to the prior discussion (this one as a candidate of what was being talked about). Consequently, it frames the action of showing as relevant to the group of ratified participants to which it is explicitly addressed ("you guys") while prefacing the showing, i.e. prospectively orienting the attention of recipients to what might appear.

As this example illustrates, showings involve not just 'seeing', but also 'seeing as'. Preliminary work, somewhat similar to prefaces to stories, frames the way objects might be recognized as part of some interactional project. Here, the deictic expression frames the image to be shown as a candidate instance of the image being discussed. In that respect, the prefatory turn is framed as a self-deprecating assessment that downgrades the potential novelty of the image to be shown. This move seems to work as a way to preempt a possible inadequacy within their larger interactional projects, since if everybody has already seen the picture, then it may not be the one they were looking for.

The subtle distinction between seeing something so that it is visually recognizable, and seeing it so that its showing fulfills a larger interactional project, can be observed in the reception sequence. Once the image is on display (Line 11), it becomes available to be scrutinized and evaluated. Recipients are expected to do just that, and they collaboratively achieve a differentiated reception (Lines 12-23). First, they orient to the mere visibility of the image on screen. ELLIOT displays visual recognition by describing explicitly what he sees (Line 14). zELMA first tries to figure out what the image is (Line 12) and then displays visual recognition (the self-interrupted turn and then laughter, line 16), without making explicit what she actually sees. ELLIOT and ZELMA are the only two participants who orient toward the interactional relevance of what they see with respect to their larger project. However, they do not see the candidate image as the one they were referring to: such a denial is implied in BLUE's negative assessments (Lines 17, 19 and 23), with which Zelda aligns (Line 21) as eventually does TUCK (Line 25). Though visually successful (an image was recognizably shown) the showing proves to be an interactional failure. COSMO, BLUE and 
TUCK dramatize this point in a collaborative flurry of joking turns that highlight such failure (Lines 25-28) before TUCK acknowledges this failed attempt by laughing and making the image disappear without further comments.

After TUCK produces yet another unsuccessful showing (section not transcribed), zELMA initiates a showing of her own.

\section{Extract 2.}

1. ZELMA: ((manipulates cell phone))

2. I have that one a ((ZELMA brings cell to the camera))

3. a Image 2.1

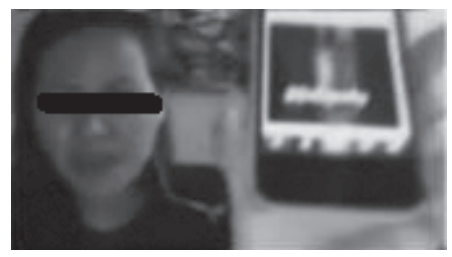

4. ZELMA: I don't even know if you can see it

5. a ((ZELMA brings cell closer to webcam))

6. $\quad$ Image 2.2

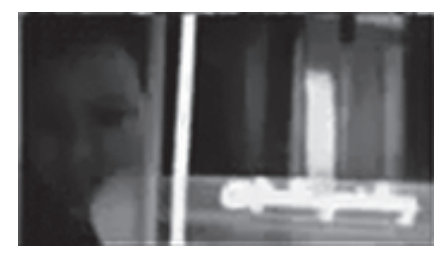

7. ZELMA: No (.) that- that is the one [( ) from the

8. ELLIOT: [Yeah you can actually ( )

9. ZELMA: = Music Box

10. ELLIOT: ((mumbles)) what was the white ( )?

[SECTION NOT TRANSCRIBED. TUCK PRODUCES A FAILED SHOWING]

ZELMA manipulates her cell phone while announcing that she has found a candidate image (Line 2), and brings her cell phone to the camera so that it becomes visible in her video frame. Unlike material objects such as zELMA's cell phone, digital objects are available online, and are therefore retrievable to all participants. Through her minimal and unmarked announcement used as a preface to the immediate showing, ZELMA displays a strong sense of entitlement to look for and show a candidate image herself. zELMA builds on the public online accessibility of the objects they are looking for as a resource to enact their search as (a) collaborative, in the sense that they can all search on their own, and (b) 'democratically'-organized, in the sense that she positions herself (and by extension all participants) as having the same right to search and show as the host. 
However, with respect to the workings behind the showing itself, participants have asymmetric resources. First, there is the issue of skills: replacing one's video feed with a digital object requires some skilled manipulations of the Hangout environments. Unlike material objects that can simply be brought to the camera (a material showing), getting a digital object on screen i.e. performing the digital showing TUCK did before, usually involves time, effort and a display of interface literacy. Consequently, this is often done as a skilled performance (see Example 2 in the next section). Here, by resorting to the material showing of her mobile phone as a next best solution and accounting for poor recognizability (her anticipatory assertion "I don't even know if you can see it", Line 5), zELMA marks her showing as different from TUCK's, and signals that she might not have the resources to do a proper digital showing. A second source of asymmetry is enacted within the digital ecology itself: while all participants can in principle replace their own video image with a digital object, only the host can ensure the image on screen is frozen in the main video window. In that sense, the Hangout host has more power than the 'guests' in the production of a digital showing.

This example brings to the fore some interesting points. To begin with, multiple layers of visual resources that ensure some form of perceptual access to the showable (e.g. use of cellphone for alternative showings) are made possible due to the digitality of the showable object. Next, this interaction highlights both a 'classic' showing, in the sense that there is one person in charge of searching for the object and putting it on display, and a more 'democratic' practice in which other participants also look for showable objects and produce alternative showings. In the following examples, we explore more collaborative work not only as part of the reception sequence, but also as central in terms of distribution and access to the showable object.

Finally, we may infer that material and digital showings have the same sequential organization, characteristic of video-mediated showings in general: (a) a preliminary sequence in which one party turns an object into a relevant showable and becomes a 'show-er', and a preface that projects the showing and frames the way the showable may be seen by its potential audience; (b) the showing itself, which involves some non-verbal manipulation of the showable and its environment; (c) the reception of the showing in which issues of visual adequacy may be separated from issues of interactional adequacy. However, material showings and digital showings differ with respect to the way participants may access the 'showable'. Material showings are mostly highly asymmetric with only one participant usually having manipulative and perceptual access to the showable. In contrast, digital showings that involve online 'showables' are usually more symmetric (with respect to manipulative access in particular) and thus support a wider range of collaborative 
practices. Nevertheless, the work of performing a digital showing also requires less equally distributed skills, and may involve power asymmetries embedded in the interface. As the next example shows, technical skills and manipulations both expand and transform the required preliminary work.

\subsection{Example 2: Screen sharing a video clip as a collaborative activity}

Through the following example we intend to highlight the emerging nature of some digital showings as the preliminary work is greatly expanded due to the practical difficulties of managing joint digital access. As we will argue, prefaces are highly collaborative, require technical skills, and enact emergent collaboration frames where participants display particular capacities, rights and obligations with respect to the showing under way. The case we discuss in this section belongs to a HOA loosely organized around the topic of cars, racing, and bikes. The extract starts with one of the participants (RAY) narrating a personal anecdote about racing one of his cars together with LIAM. As evidenced by RAY (Lines 1-2), the event in question was recorded and uploaded to YouTube. RAY's narrative is the motivation for the subsequent showing. Figure 3 below shows all participants. RC is the Hangout host.

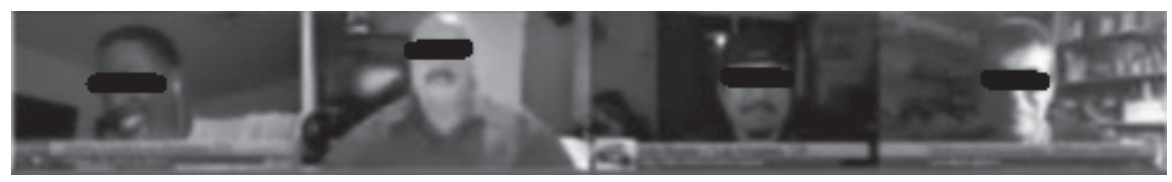

Figure 3. RAY, RC, LIAM, and GREC

\section{Extract 1.}

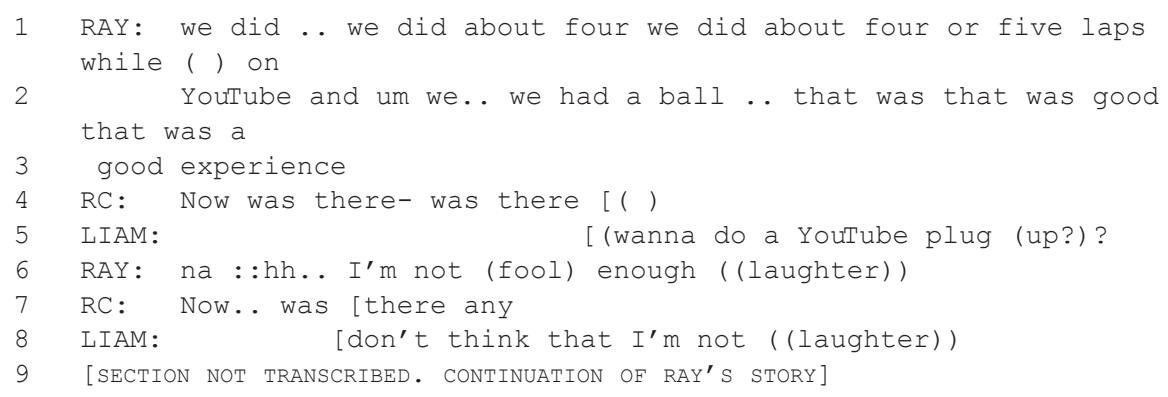

As is the case with video-mediated showings of physical objects ('material showings'), a preliminary step within the sequential organization of digital showings consists of making the showing a relevant and joint concern. In Extract 1 above, 
RAY tells a story about a prior car driving experience that was recorded on YouTube (Lines 1-2). His story therefore includes a reference to a digital object, potentially accessible, retrievable and shareable online. This enacts the relevance of a showing, and LIAM orients to that possibility by asking whether he would like to show the video in question (Line 5). More generally, talking about an object in a way that stresses the fact that it is easily available and 'showable' effectively projects a potential showing as a relevant next move. Let us also remark that this reference frames what the showable is and how, should it ever be shown, it might be viewed, i.e. as a video recording of the very experience RAY is narrating.

\section{Extract 2.}
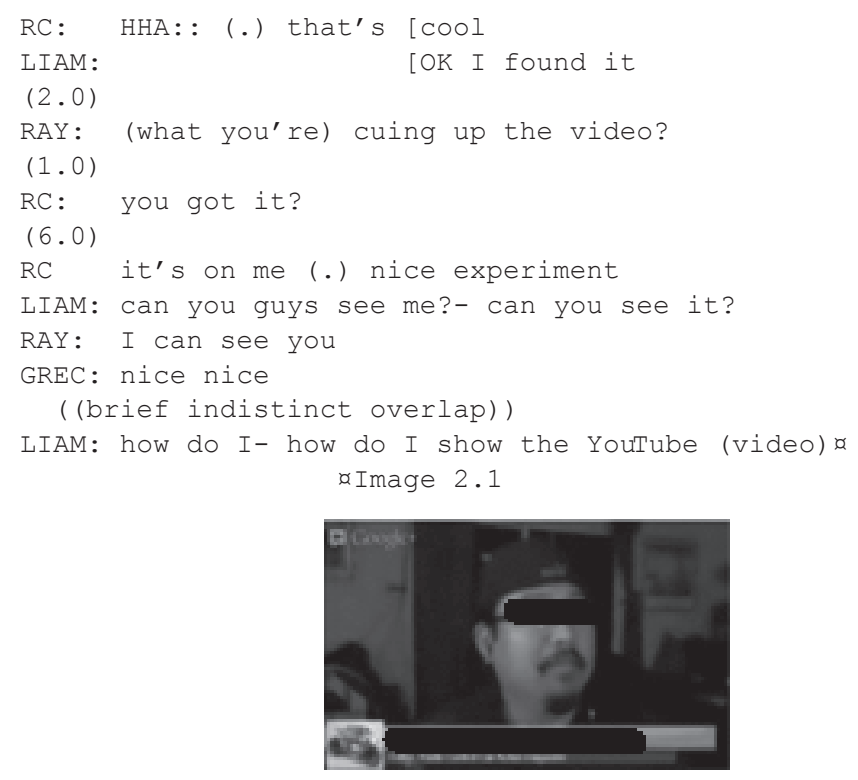

RC: mmm let me: (.) hold on one second $(4.0)$ a

Image 2.2

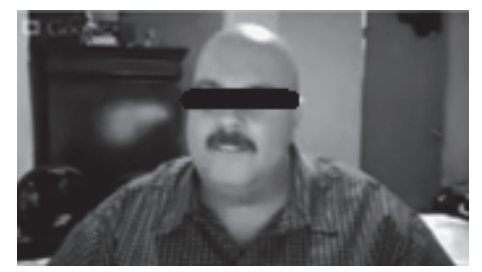

18 RC: u::hm (are we gonna) share video?

19 LIAM: yeah

20 RAY: yeah

$21 \quad(1.0)$ 
After participants have evoked the possibility of showing the video, RAY goes on telling his story (section not transcribed). RC, the host, provides a positive assessment (Extract 2, line 1). Quasi-simultaneously, LIAM produces the overlapping announcement "OK I found it" (Line 2). The design of the announcement highlights the relevance of accessing and showing the video. First, the OK in initial position suggests the announcement is oriented retrospectively toward some prior concern, so that actually finding the video and making it public was relevant. Second, the intelligibility of the indexical "it" as a reference to the video and the relevance of accessing and showing it are mutually constitutive. The other participants respond by orienting to this announcement as projecting a showing. RAY asks whether LIAM is preparing to show the video (Line 4), and after a check, the host assesses the possible showing as a "nice experiment" (Line 8).

Let us remark that, as is often the case with digital showings, these responses emphasize the technical work involved in actually doing the showing (RAY's reference to "cuing up the video, line 4) and even frame the showing as a skilled performance (RC's notion of it being a "nice experiment"). Similarly, LIAM's check, "Can you see me? Can you see it?" (Line 9) displays two things: (a) that LIAM is actually running the video they were talking about, which maximizes the imbalance with respect to access, and (b) that LIAM displays a distinctive stance with respect to such imbalance: he is to give access to the showable and to share the running video so that it can be jointly experienced. This is the sense of 'showing as sharing' that emerges here, which involves the minimization of perceptual imbalance with respect to access.

After getting negative responses (Line 10), LIAM finally states his inability to produce a showing sequence (Line 13). In a context in which showing/sharing is a relevant interactional project, such a statement is hearable as a request for help, and the host (RC) displays precisely such an understanding. RC instructs the others to hold on (Line 15) and utters a confirmation polar question "are we gonna share the video?" (Line 18) aiming for a collective ratification of their current project 'showing as sharing', which others confirm (Lines 19-20). Coming after LIAM's statement of inability, RC's intervention can be seen as a claim to take charge and lead the required work, framing himself as the potential future show-er. This makes relevant an emergent collaborative organization of work with respect to their ongoing project. At that moment, LIAM has the video running on his computer, RC tries to work with LIAM to get the video running on his computer instead, so that it is visible to all. RAY and GREC, on the other hand, assume a position of onlookers with respect to this collaborative work. Such emergent collaborative work is displayed in the following extracts. 
Extract 2. (continued)
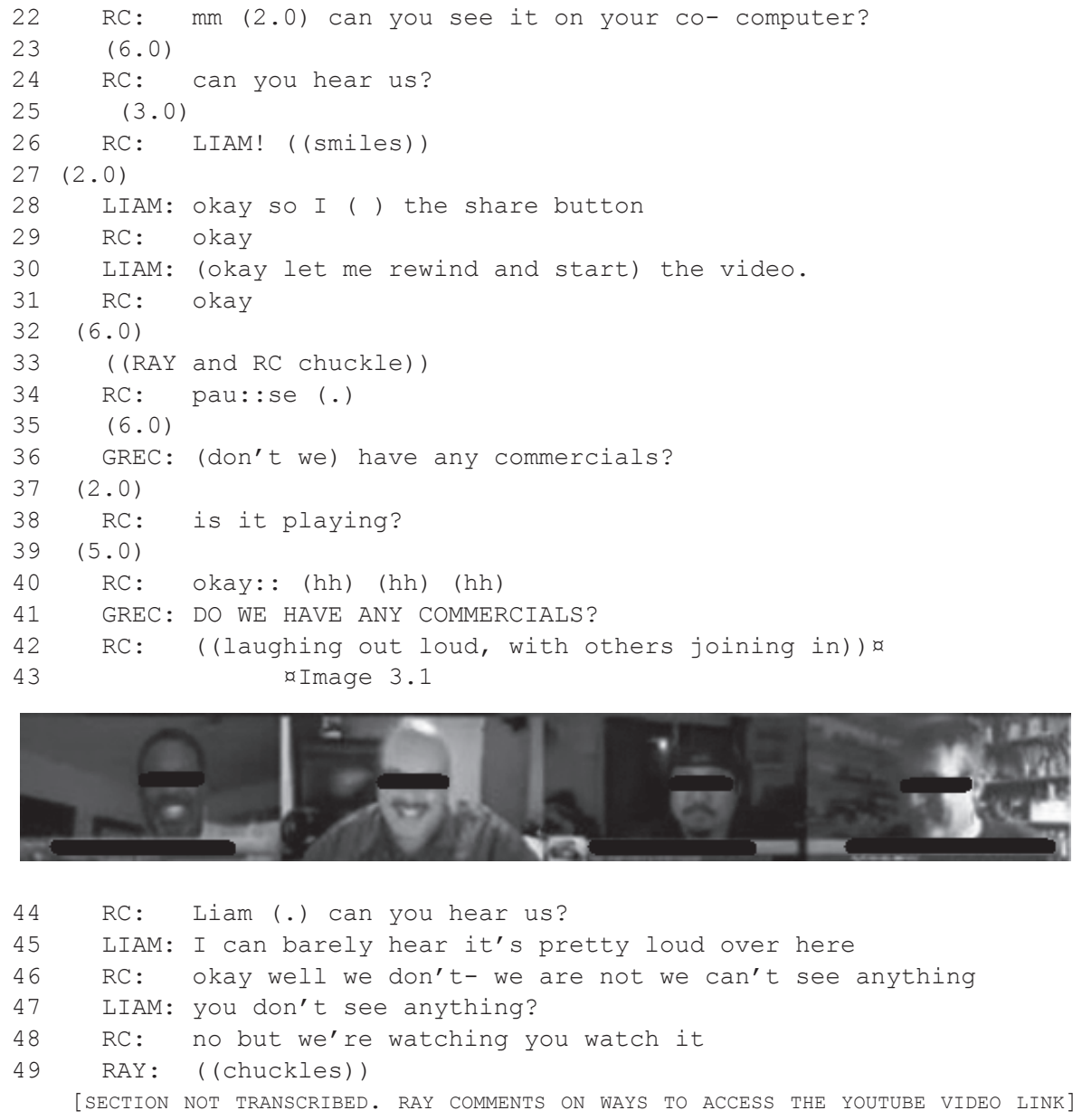

The excerpt above displays the collaborative organization within the Hangout participation framework. As we can see, RC's turns are mostly addressed to LIAM. First, RC summons LIAM's attention (Lines 22-26), indexing his ratified role in the collaboration framework, i.e. organizing the eventual sharing of the video. Moreover, all of RC's and LIAM's turns orient toward the video sharing project in some way. Conversely, GREC and to a lesser extent RAY are mere onlookers and self-select as ratified participants at possible turn transitions (e.g. GREC in line 41). However when GREC does so, it is to joke about his status as an onlooker since the joke itself refers to a passive form of viewing, i.e. viewing commercials.

Because their project is to share a video in the shared interactional space, a particular tension between two ways of engaging visually with what appears on their screen is highlighted. That is, engaging with what is visible on screen as a resource 
that can be made relevant to their ongoing interaction on the one hand; and on the other, viewing what is on screen as a spectacle. This particular tension surfaces in lines 46-49. In line 46, RC states that they cannot see anything, which is hearable as a negative status report with respect to their ongoing project. LIAM responds with an interro-negative visual check, projecting a no answer (Line 47). RC provides a type-conform negative response (Raymond 2003) together with an elaboration "but we're watching you watch it" (Line 49). This response intensifies ironically their interactional trouble. First, it acts as a reminder of the maximal asymmetry between LIAM and the rest. Second, by describing themselves as watching LIAM watch the video, RC highlights LIAM's apparent disengagement from the interaction, which turns the others into onlookers. The irony lies in the misplacement of this interaction-generated categorization. Because of LIAM's behavior, the other three participants become onlookers with respect to the 'wrong' type of screen (LIAM's face as he watches the video in question).

GREC humorously topicalizes this disengagement by evoking the traditional use of commercials on TV as a way to kill time whenever technical difficulties occur. This not only marks the participants' momentary role as onlookers but also points to the possibility to resort to the embedded frame in mediated communication by overtly making reference to the mediated nature of the interaction. It has been suggested that handling VMC requires participants to engage in parallel activities, since they advance the ongoing conversation while managing the technical aspects of the medium. This double engagement prompts interactants to make public their parallel focus of attention, topicalize their technical manipulations, and also offer other participants the possibility to align themselves in the new focus of attention (Heath \& Luff 1992).

The process of trying to share the video goes on for several minutes, too long for us to analyze in detail. However, even at this stage we can already see how the preliminary work expands into a complex collaborative and highly emergent process. We will now move directly to the final stages of the showing, when the technical problems are about to be solved.

\section{Extract 3.}

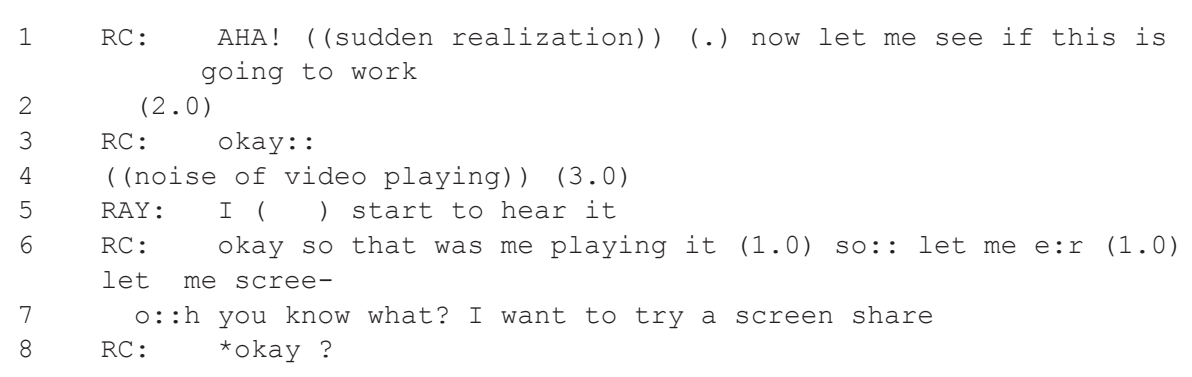


its change-of- stake token in initial position, is indication that the previous technical problems might be solved. Furthermore, RC's turn is hearable as a preface to the showing. Indeed, RAY seems to hear it as such by providing a cue that he hears the video, thus giving feedback to RC's projected action. Due to the imminence of a video showing, 'just hearing' is taken as an indication that the video cannot be seen and thus, that the projected showing did not work. RC hears it that way since he accounts for that comment retrospectively (Line 6), framing his previous turn as an occasioned announcement, rather than the actual preface to the showing. RC then goes on to produce a preface ("I want to do a screen share", line 7), and marks the sequential position to secure a go ahead ("okay?", line 8) which he gets (Line 9).

The preface works to open up a slot of focused expectation for the projected action, in which participants are supposedly orienting visually toward the screen until a recognizable 'showable' appears. While prefaces to stories have been argued to preserve the obligation for recipients to listen while inhibiting the usual turn-taking system for ordinary conversation (Sacks 1992), prefaces to showings seem to do something similar with respect to the visual field, i.e. securing the recipients' attention into a visual domain where the potential showing is to appear. Prefaces also constrain the production of turns-at-talk at that particular juncture, with turns that orient to the showing itself being hearable as relevant, and those which do not, as indices of potential disalignments. Evidence that RC's recipients align with the sequential implications of his preface is provided by the long silence (Line 10), followed by RAY's negative statement ("nothing", line 11), which marks such an event as missing, both practically and sequentially, as well as LIAM’s similar initial turn-construction unit (Line 14).

However, in the meantime, the video does appear on screen and LIAM self-corrects to provide a noticing of that event ("there it is", line 14). The showing reception involves seeing the showable, in the sense of detecting a visual event, as well as recognizing it as the kind of showable that has been framed during the preliminary work. In that respect, LIAM's noticing in line 14 is highly generic and indexical (the use of 'there' and 'it'), ensuring only that an event has occurred, that a possible 'candidate showable' has appeared. The 'show-er' (RC) then provides a visual confirmation check, "see it" that is still generic and indexical but makes explicit the visuality of the event (Line 15). Although RC gets a confirmation from GREC (Line 18), and a technical suggestion from LIAM (Lines 19-20), he issues another confirmation check line ("do you guys see it?", lines 21-22) probably fishing for more acknowledgements from less peripheral participants. ${ }^{3}$ GREC confirms, and LIAM provides a thumbs-up gesture (Line 24, Image 3.4). RC displays understanding

3. RC acts as the host and the show-er, LIAM has access to the video, and both LIAM and RAY were racing the car when the video was taken. 
that the showing is practically and visually successful by providing an assessment of the content of the video itself (Line 27).

We find again an overall organization of the showing sequence, which we had started to identify in the previous section, involving the following:

- A preliminary sequence that starts when a showing emerges as a relevant interactional project. This preliminary phase usually ends with a preface that provides an orientation for recipients to watch, as well as a slot for them to give a 'go ahead', which enacts a form of conditional relevance with respect to the talk that might unfold during the showing.

- Expansions of this preliminary sequence and elaborations of the collaborative work aimed at displaying the image (often involving multiple checks highlighting the emergent character of the process).

- The actual production of the showing in which some object is made visually available.

- The acknowledgement of the showing itself in which participants demonstrably deal with two different concerns: that they have both visual access to a recognizable 'showable' (which marks the success of the showing in what we will call ostensive terms) and that they are able to view it with the kind of outlook in which it was initially framed (to 'see as' as opposed to just 'see').

The fact that this constitutes a very particular type of digital showable surfaces in different ways. To begin with, as in the previous section, the preliminary work is considerably expanded and done as a skilled performance of individual and collective computer literacy. This is done within the emergent organization of collaboration where participants may enact situated roles and display related entitlements, rights and obligations. Second, because it is video, there is a sense of showing as sharing, where the point is not just to make the video visible, but also to run it so that it is a source of events that all may experience at the same time (or rather simultaneously enough with respect to all practical purposes). Last, because of the performance-like character of the showing and its technical uncertainties, the reception of the showing is also expanded, and seems to proceed in a stepwise way. In this sense, participants collaboratively and successively ascertain that they can see and recognize a showable, that the showable can be viewed by all as a running source of noticeable events, and finally, that they may mutually orient to it according to the initial framing of the showing project. 


\subsection{Example 3: Link-shares and the multiple layers of viewership}

We now turn to a sequence in which participants refer to an image, which would be understood as a preface to a showing, without eventually displaying it in the shared main video window. In HOAs, sharing an image involves making it public with respect to a wider audience. The participants of this Hangout agree that the content of the image in question is unsuitable for such publication due to Google+ policies (Google 2014). This points to how showings in HOAs are embedded in a complex moral economy, which articulates the organization of interaction and larger scale institutional policies. Moreover, as we will see, showing the image remains relevant enough in the interaction for participants to elaborate a different way to view, share and talk about the image. Eventually, the showing is carried out by sharing the link to the image. Through this example, we wish to highlight the notion of a multi-layered viewership and of a particular form of showing: viewing privately while experiencing jointly. We will argue that this type of link-sharing activity effectively 'counts' as showing. The main video window is set on one of the participants (gm4) during the whole interaction under analysis (Image 1.1, Extract 1 below). Figure 4 shows all participants. dusty, in the middle, is the Hangout host.

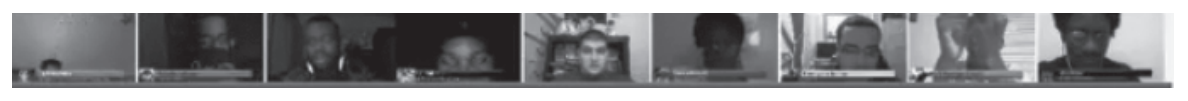

Figure 4. GM1, GM2, BROD, GM4, DUSTY, GM5, GM6, GM7, and PROF

\section{Extract 1.}

GM7: ((Video main window set on GM4)) we- we got we got some time we got

a Image 1.1
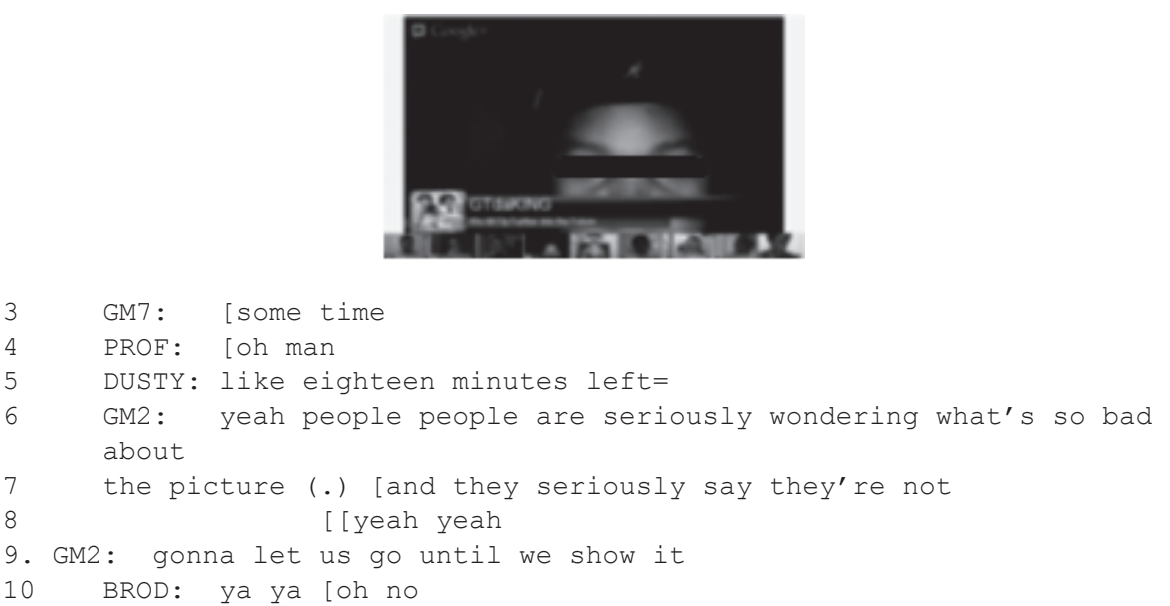
12 GM2: okay no someone's got-a- I might've seen this photo before but someone

13 has to show me something like a-

14 GM5: no this j-just upload it to my [ ( )

15 PROF: [no no no no no no no no Brod is

Brod is

16 gonna send it to me and I'm gonna take a screen shot

17 ((DUSTY raises both hands))

18 BROAD: yeah yeah

19 GM6: I wonder if (that's) I'm looking for mine's right now ['cause I ( )

We join the action as participants talk about how much time they have left in the stream (Lines 1-3).GM2 then produces a statement referring to a picture they have been discussing (Lines 6-9). The category "people" here refers to the Hangout audience and to their LTCs. This move makes relevant a membership categorization device "us" as in us, the ratified participants in the video stream, and "them" or "people" as the active viewing audience that posts LTCs. Comments from viewers come in rapidly and most of them revolve around the image in question. Due to space limitations, we only include one screenshot of some of the messages around line 6 (Figure 5 below).

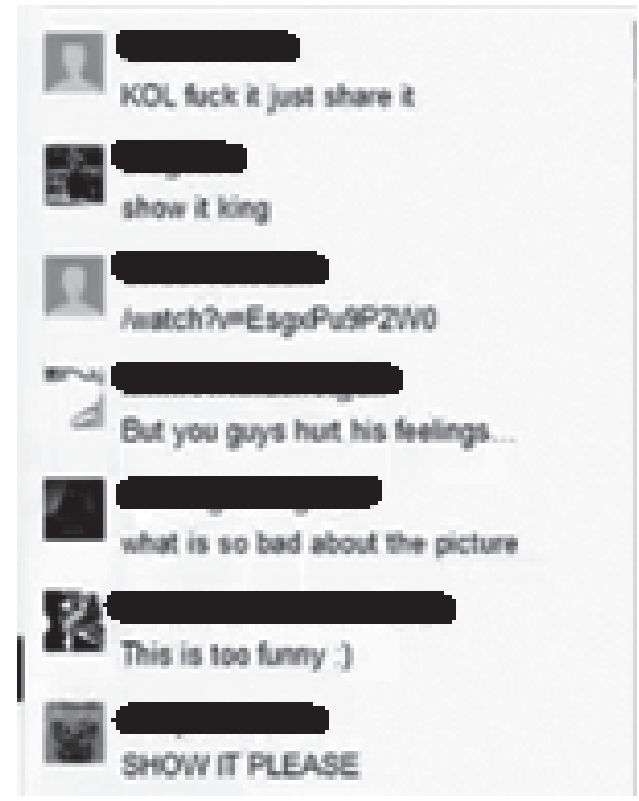

Figure 5. Live Text Commentaries 
Several of the LTCs involve questions about the picture they have been discussing (e.g. "what is so bad about the picture", "who is on that picture again?" as well as explicit requests to show it, such as "show it", "show it (name)", with a somewhat increasing urgency "SHOW IT PLEASE", "KOL fuck it just share it". By acknowledging these requests in a reported discourse format, GM2 turns the viewers' expectation to see the image into a relevant topic (Lines 15-16).

Through multiple responses, participants orient to the showing as a collaborative project, some do this by describing the kind of individual access they have; and others, by describing how they might show or share the image. First, GM2 states the type of access he has to the image (he might have seen it before but does not have visual access to it now) and requests some help in securing access (Lines 12-13). GM6 indicates he might be looking at it right now (Line 19). GM5, on the other hand, requests for it to be uploaded to an accessible place (Line 14), perhaps already planning to show it to the others. PROF understands it this way by sketching another method to show and share the image: one of the participants, BROD, will send him the image so that he can take a screen shot. Ratified participants thus display equal rights to see the image (their entitlement to request help in getting access to the image when they do not have it) and equal entitlement to propose ways of sharing the image. Only the host (DUSTY) has special rights and obligations. However, since none of the participants is implicated in the image (as were RAY and LIAM in Example 2), the relevance of the showing project enacts them as a 'democracy' of viewers and potential contributors to the showing as a collaborative achievement. This is also only possible because the 'showable' is a digital object, potentially retrievable from the web by all of them, though as in all of our examples the actual showing requires some specific computer skills.

\section{Extract 1. (continued)}

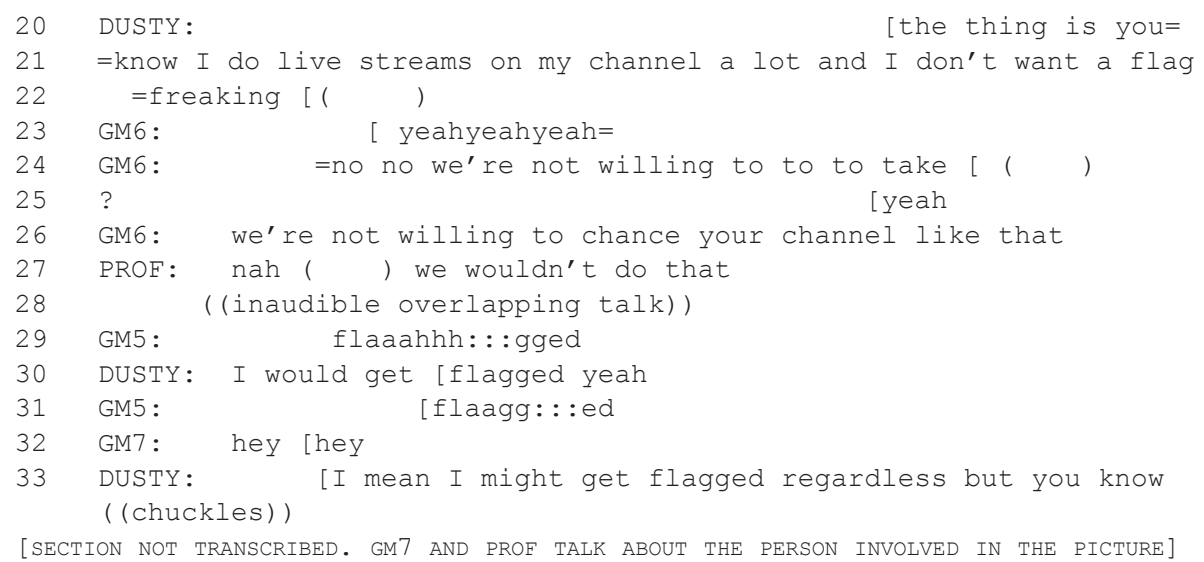


DUSTY orients to PROF's previous turn as a pre-sequence, an announcement that projects a sharing. The next sequential slot is used to voice concerns about the propriety of such a sharing in terms of the potential sanctions that might be applied to DUSTY's channel ("be flagged", lines 21 and 22). DUSTY makes relevant his special responsibility as the host (and the membership categorization device 'host' and 'guests', i.e. the ratified video participants in the channel). By doing this, DUSTY also invokes some hypothetical institutional Google-based moderators as a resource to stifle the emerging 'democratic' collaborative project of showing. DUSTY and the other participants display awareness that their public stream is embedded into a wider web of institutional constraints, so that their activities in general, and showings in particular, are embedded into a complex moral economy of visibility. Consequently, the reference to institutional norms and possible sanctions is used as a resource to revise the current interactional project.

The other participants orient both to this categorization and to the sequential implications of DusTy's turn as a 'block' with respect to the projected showing. They provide a volley of supporting turns (Lines 23-28) in which they enact themselves as a "we", the video guests (Lines 24, 26 and 27), supporting DUSTY's position and displaying their solidarity with DUSTY's concerns as well as their own cohesion as guests. DUSTY uses this display of support to more strongly reformulate the possible sanctions, going from his initial "I don't want to get flagged" to "I would get flagged yeah" (Line 30). DUsTy displays here his understanding that sharing the image is not a relevant project anymore. Yet, co-participants will still orient to the visual relevance of the image with respect to their discussion while aligning with the rejection of the sharing. As we will see in the extract below, this orientation is done by topicalizing their efforts to retrieve the image to access it individually.

\section{Extract 2.}

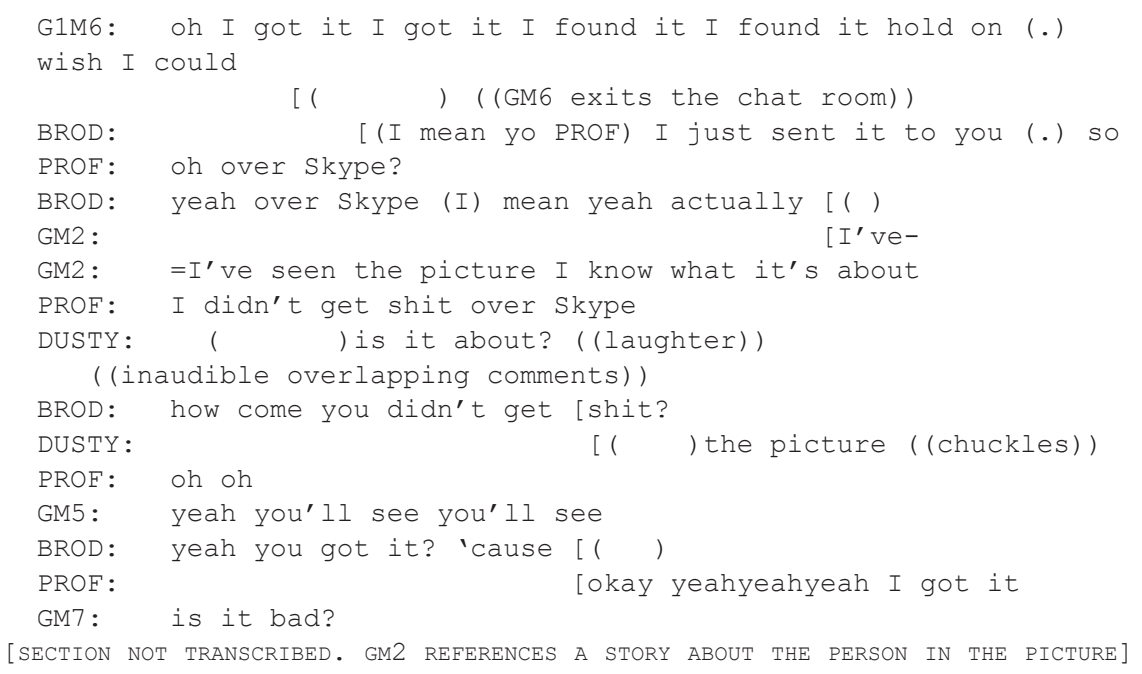


The extract above shows the actual sharing of the content outside the Hangout platform. GM6 announces he has found the picture (Line 1), which is followed by вROD stating he had already sent the picture to Prof over Skype (Line 3). Participants thus make public the event of getting access to the image, and the collaborative work of providing access to a co-participant, as evidenced in the exchanges between BROD and PROF. Simultaneously other participants display that they do not have access to the image, but still refer to it in a way that reinforces its status as the current relevant issue, such as DUSTY asking about the picture (Line 9) or GM2 stating he has already seen it (Line 7). Consequently, there is at that moment a somewhat multi-layered and partially fragmented participation frame in which participants orient toward the image in different ways, with some about to or already having access to it, while others still do not.

As the extract below shows, PROF's actual reception of the image changes the context and enacts a shift in the orientation of all co-participants.

\section{Extract 3.}

BROD: so you go it?

PROF: hold on (.) just one second

GM5 : damn. .

BROD: you gotta ( ) so

$(4.0)$

PROF: O:: :::H YOU CAN'T SHOW THIS BRO!

( (multiple laughter))

GM5 [yeah I told you you can't show that you can't show that

(hh)

(hh)

BROD [I told you ((overlapping laughter))

BROD I told you [I told you you can'show something like that

GM2

[ (ingrown) link it to (something)

DUSTY: link link it to my skype man (.) link it to my skype man

PROF: [that is terrible (.) oh my

[( (overlapping loud laughter))

PROF: terrible

GM5: it is terrible man ( )

BROD: yeah ( ) I told you, I told you that shit was bad son.

you can't link

19 I can't. you can't show this shit ((laughter))

20 ((multiple laughter))

21 DUSTY: OK, let me see this

( (multiple laughter and response cries as GM1, GM2

DUSTY, and GM7 see the

picture))

GM2: OOOH!! ((loud laughter))

GM7: oh my GO:::D! ((loud laughter))

GM2: OH: : : SHI : : t !

PROF: ((chuckles)) yeah you can't show that

GM2: no no no

((multiple laughter and overlapping comments)) 


\section{Laura Rosenbaun and Christian Licoppe}

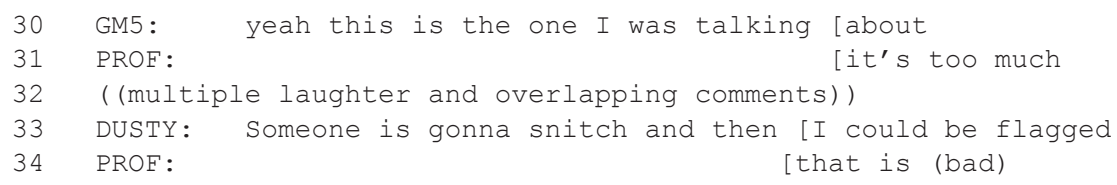

BROD has sent the picture to PROF via Skype (building on the digitality of the object). Their collaborative activity is sequentially organized as showing by link-sharing, with BROD as a show-er having announced and initiated the showing. As signaled in Extract 2 line 3, the showable was framed as being too transgressive to be shared. In this sense, the image is framed so that PROF does not only have access to it (the ostensive purpose of the showing) but sees it as something too harsh or graphic to become public (the interactional purpose of the showing). Along the same line, there is first a visual access-checking between BROD and PROF (Lines 1-4) and then a crucial turn by PROF marking his viewing of the image "O:::::H YOU CAN'T SHOW THIS BRO!" (Line 6). This turn is marked as occasioned with a change-ofstate token, and in this context the occasion is PROF's opening the image. PROF's assessment confirms not only that he sees the image, but that he sees it in the way it was initially framed, that is, as something that cannot be made public.

PROF's turn (Line 6) enacts a new framework in which just talking about the image is now not enough to be a ratified participant. First, GM2, previously content with having already seen the image, now changes stance and requests access to the image here and now ("come on somebody just link me this damn thing", line 10). GM2 displays his understanding that the pre-requisite to contribute to the interaction is not just having viewed the image sometime in the past, but rather to experience it as the interaction unfolds so as to be able to appreciate it publicly and jointly. This shift is amplified by PROF's repeated re-enactments of his initial response, with BROD and GM5 affiliative second assessments (Pomerantz 1984) in reply (Lines 14-29). This seems to draw DUSTY into the fray, for he asks to see the image as well (Line 13). Co-participants' emphatic references to an object they do not see but might have access to makes such access a potential concern to all participants, even those out of the visual loop.

Participants' orientation now involves individual viewings of the image, yet done in a public way so that the experience is joint. This is the next best thing to actually displaying the image, and points toward the interactional value of publicly and collectively experiencing the digital object while viewing it individually. This emergent reception is displayed in the way they publicize their efforts to get access to the image and whether they have access or not, and even later as the talk evolves into a chorus of response cries, occasioned assessments and laughter (particularly lines 32-41). This type of showing as a concerted viewing is a complex collaborative accomplishment, the very possibility of which relies on the digitality of the object and the way in which it can be retrieved and made available in fragmented ecologies (Luff et al. 2003). 


\section{Conclusion}

We have discussed a set of showing practices characteristic of digitally embedded video-mediated settings. Such showings involve participants' collaboration to retrieve a digital object previously made relevant in the interaction, and to which some may already have access. Through collaborative computer-based procedures, participants achieve a shared perceptual experience of these objects on screen. Compared to 'material showings', in which participants bring either some material feature of their environment to the camera or the camera to a given feature, we found 'digital showings' to be both similar and unique.

In line with glossing these practices as 'showings', we found they share a similar sequential organization involving: (a) preliminary work, in which reference to a particular object as a 'showable' enacts the relevance of its showing, usually initiated through a preface that frames the way the object should be seen; (b) the showing itself, in which the object is manipulated by the show-er into a joint perceptual field, opening a slot for viewers to respond; and (c) viewer's response and assessment displaying perceptual access and a stance with respect to seeing it the way it was framed to be seen.

While sharing an overall sequential organization with 'material showings', digital showings are distinctive in that accessing the object and getting it on the screen requires special collaborative work and technical skills that may be asymmetrically distributed and enacted on a moment by moment basis as the interaction unfolds. Because of this, the pre-showing sequence expands into a complex collaborative performance in which, and through which, participants display different participation statuses and deontic stances. Furthermore, keeping the object on the screen also requires technical manipulations. From the reception end, participants need to confirm they are seeing the object and ratify viewership. Several times, the 'shower' offers slots for others to agree, view, and evaluate the showable. Moreover, the repetition of such invitations for reception may signal some uncertainty with respect to its process.

Our data suggest three crucial dimensions to these digital showings. First, when one participant retrieves an object to show to others, the showing can be done by alternative paths engaging different modes of collaboration, such as visually displaying the object on the video window (screen sharing it) or by making it accessible to viewers by means of sharing a link to that digital object so that every ratified participant may access it independently yet jointly (Example 3 ). Such collaboration unfolds in a multi-layered ecology of visual resources (e.g. cellphones or other devices as alternative displays, use of platforms such as Skype to share links). Second, claims and obligations regarding the objects can be subtly distributed. Unlike material showings in VMC, the person displaying the image (in a screen sharing mode) is not necessarily the 'owner' of that object or the person 
with the strongest ownership claims. This is evidenced when the latter allows and even mandates another participant to share the object (as with the video in our second example). Entitlements to specific forms of collaboration may be displayed and enacted by all participants in an emergent fashion in the course of the collaborative process of showing/sharing a digital image or video. Last, and particularly for these live, public video chats, there is a fragmentation of reception and a multi layering of viewership (hosts, participants with differing viewership status at a given time, online audience potentially responding through LTCs). All this suggests that understanding the practices that may conveniently be glossed as 'showings' might be as crucial to understand the anchoring of video-mediated sociality in its multi-layered, fragmented ecologies as the study of pointing practices has been to show the embodied character of co-present human communication and its anchoring in a material setting.

\section{References}

Aaltonen, T., I. Arminen, and A. Raudaskoski. 2014. "Photo Sharing as a Joint Activity Between an Aphasic Speaker and Others." In Interacting with Objects: Language, Materiality, and Social Activity, ed. by M. Nevile, P. Haddington, T. Heinemann, and M. Rauniomaa, 125-144. Amsterdam: John Benjamins, 125-144.

Buhler, T., C. Neustaedter, and S. Hillman. 2013. "How and why Teenagers Use Video Chat." Proceedings of the 2013 ACM Conference on Computer Supported Cooperative Work, 759-768.

Clark, H. 2003. "Pointing and Placing." In Pointing. Where Language, Culture and Cognition Meet, ed. by S. Kita, 243-268. Hillsdale NJ: Erlbaum.

Chovanec, J., and A. Novaka. 2010. "Online Discussion and Interaction: The Case of Live Text Commentary." In Cases in Online Discussion and Interaction, ed. by Leonard Shedletsky, and Joan E. Aitken, 234-251. Hershey: IGI Publishing. doi:10.4018/978-1-61520-863-0.cho12

Goodwin, C. 2000a. "Action and Embodiment within Situated Human Interaction." Journal of Pragmatics 32 (10): 1489-1522. doi:10.1016/S0378-2166(99)0oo96-X

Goodwin, C. 2000b. "Practices of Seeing. Visual Analysis: An Ethnomethodological Approach." In Handbook of Visual Analysis, ed. by T. van Leeuwen, and C. Jewitt, 157-182. London: Sage Publications.

Goodwin, C. 2003. "Pointing as Situated Practice." In Pointing. Where Language, Culture and Cognition Meet, ed. by S. Kita, 217-241. Hillsdale NJ: Erlbaum.

Goodwin, C. 2013. "The Co-operative, Transformative Organization of Human Action and Knowledge." Journal of Pragmatics 46 (1): 8-23. doi:10.1016/j.pragma.2012.09.003

Google, 2014. User Content and Conduct Policy. Retrieved from http://www.google.com/+/policy/ content.html

Heath, C., and P. Luff. 1991. "Disembodied Conduct: Communication through Video in a MultiMedia Office Environment." Proceedings of the SIGCHI Conference on Human Factors in Computing Systems 99-103. 
Heath, C., and P. Luff. 1992. "Collaboration and Control: Crisis Management and Multimedia Technology in London Underground Line Control Rooms.” Computer Supported Cooperative Work (CSCW) 1 (1-2): 69-94. doi:10.1007/BFo0752451

Heath, C., and D. Von Lehn. 2004. “Configuring Reception. (Dis-)Regarding the 'Spectator' in Museums and Galleries.” Theory, Culture \& Society 21 (6): 43-65. doi:10.1177/0263276404047415 Heritage, J. 1984. "A Change-of-State Token and Aspects of its Sequential Placement. Structures of Social Action." In Structures of Social Action, ed. by J. M. Atkinson, and J. Heritage, 299-345. Cambridge: Cambridge University Press.

Kita, S. 2003. Pointing. Where Language, Culture and Cognition Meet. Hillsdale NJ: Erlbaum.

Licoppe, C., and J. Morel. 2012. "Video-in-Interaction:"Talking Heads" and the Multimodal Organization of Mobile and Skype Video Calls." Research on Language \& Social Interaction 45 (4): 399-429. doi:10.1080/08351813.2012.724996

Licoppe, C., and J. Morel. 2014. "Mundane Video Directors in Interaction: Showing one's Environment in Skype and Mobile Video Calls." Studies of Video Practices: Video at Work, 135-160. London: Routledge.

Litt, E. 2012. “Knock, Knock. Who's there? The Imagined Audience." Journal of Broadcasting \& Electronic Media 56 (3): 330-345. doi:10.1080/08838151.2012.705195

Luff, P., C. Heath, H. Kuzuoka, J. Hindmarsh, K. Yamazaki, and S. Oyama. 2003. "Fractured Ecologies: Creating Environments for Collaboration.” In Human Computer Interaction, 51-84. doi:10.1207/S15327051HCl1812_3

Mondada, L. 2011. "Understanding as an Embodied, Situated and Sequential Achievement in Interaction." Journal of Pragmatics 43 (2): 542-552. doi:10.1016/j.pragma.2010.08.019

Mondada, L. 2013. "Embodied and Spatial Resources for Turn-Taking in Institutional MultiParty Interactions: Participatory Democracy Debates." Journal of Pragmatics 46 (1): 39-68. doi:10.1016/j.pragma.2012.03.010

Nissenbaum, H. 2009. Privacy in Context: Technology, Policy, and the Integrity of Social Life. Stanford: Stanford University Press.

O'Hara, K., A. Black, and M. Lipson. 2006. "Everyday Practices with Mobile Video Telephony." Proceedings of the SIGCHI Conference on Human Factors in Computing Systems, 871-880. doi: $10.1145 / 1124772.1124900$

Oviatt, S. 1999. "Ten Myths of Multimodal Interaction." Communications of the ACM 42 (11): 74-81. doi: $10.1145 / 319382.319398$

Pomerantz, A. 1984. "Agreeing and Disagreeing with Assessments: Some Features of Preferred/ Dispreferred Turn Shapes." In Structures of Social Action, ed. by J. M. Atkinson, and J. Heritage, 57-101. Cambridge: Cambridge University Press.

Raymond, G. 2003. "Grammar and Social Organization: Yes/No Interrogatives and the Structure of Responding." American Sociological Review 939-967. doi:10.2307/1519752

Rintel, S. 2013. "Tech-Tied or Tongue-Tied? Technological versus Social Trouble in Relational Video Calling." 2013 46th Hawaii International Conference on System Sciences (HICSS), 3343-3352. doi:10.1109/HICSS.2013.512

Robles, E., J. Raclaw, and S. M. DiDomenico. 2016. "Mobile Phones as an Interactional Resource in Assessment Activities." (forthcoming).

Rosenbaun, L., S. Rafaeli, and D. Kurzon. 2016a. "Participation Frameworks in Multiparty Video Chats: Cross-Modal Exchanges in Public Google Hangouts." Journal of Pragmatics 94: 29-46. doi:10.1016/j.pragma.2016.01.003 
Rosenbaun, L., S. Rafaeli, and D. Kurzon. 2016b. "Blurring the Boundaries Between Domestic and Digital Spheres: Competing Engagements in Public Google Hangouts." Pragmatics 26(2):291-314.

Sacks, H. 1992. Lectures on conversation, ed. by G. Jefferson. Oxford, UK: Blackwell.

Watson, R. 1997. 'Some General Reflections on 'Categorization' and 'Sequence' in the Analysis of Conversation." In Culture in Action. Studies in Membership Categorization Analysis, ed. by S. Hester, and P. Eglin, 49-75. Washington: International Institute for Ethnomethodology and Conversation Analysis \& University Press of America.

Weilenmann, A., and C. Larsson. 2001. "Local Use and Sharing of Mobile Phones." In Wireless World: Social and Interactional Aspects of the Mobile Age, ed. by B. Brown, N. Green, and R. Harper, 92-107. London: Springer-Verlag.

\section{Authors' addresses}

Laura Rosenbaun

199 Aba Khoushy Avenue

Mount Carmel, Haifa

Israel

laura.rosenbaun@gmail.com

Christian Licoppe

I3-SES, CNRS, Télécom ParisTech

Université Paris-Saclay, 75013, Paris

France

christian.licoppe@telecom-paristech.fr 